\title{
CORRESPONDENCE
}

\section{SCULCO'S TREATMENT}

To the Editor of The British Journal of Ophthalmology

SIR,-Experience of Sculco's method of treating trachoma may be of some interest to ophthalmic surgeons. An abstract of Dr. Sculco's report to the Academy of Medicine; Naples, was published in the Journal of December, 1919. Through the courtesy of the editor of La Clinique Ophtalmologique, I was able to get four samples of the remedy from Dr. Sculco. It is a dark green powder which is to be shaken on the conjunctiva daily, kept there for one or two hours and then washed out.

I tried it on three patients in hospital, two well-developed cases, and one recent. One eye in each case was treated with copper sulphate and irrigation and the other with Sculco's remedy for ten to twenty days. The result in each case was that right and left eyes improved to an apparently identical extent, i.e., they were cleaner and less inflamed but the granulations remained unchanged. Pain, chemosis and increased discharge are to be expected in using the remedy but none of these was present in my patients. It may be that the powder had deteriorated on account of the heat.

Yours truly,

BASRAH.

P. MCRitchie.

\section{THE ESTIMATION OF REFRACTION WITHOUT A CYCLOPLEGIC}

To the Editor of The British Journal of Ophthalmology

SIR,-At the recent Congress of the Ophthalmological Society of the U.K. the champions of cycloplegic and non-cycloplegic estimation of refraction were in friendly conflict. It was suggested by the champions of the latter method that for the performance of retinoscopy at the macula it is necessary for the surgeon to use his right eye for the patient's right eye, and his left eye for the patient's left eye in order that distant fixation may be obtained. Mr. Harrison Butler went so far as to say, I gathered, that estimation under homatropin might show a lower reading of hypermetropia than does the subjective test at the types without homatropin. I agree whole-heartedly with the statement that owing to differences between the refraction at the macula and at 